\title{
Which is the best method in clinical practice for assessing improvement in vascular endothelial function after successful smoking cessation - Flow-mediated dilation (FMD) or reactive hyperemic peripheral arterial tonometry (RH-PAT)?
}

\author{
Toru Kato ${ }^{1}$
}

Received: 29 July 2020 / Revised: 20 August 2020 / Accepted: 21 August 2020 / Published online: 22 October 2020

(c) The Japanese Society of Hypertension 2020

I read with great interest the article by Fukumoto et al. titled "Predictors of the effects of smoking cessation on the endothelial function of conduit and digital vessels." The authors demonstrated a significant improvement in endothelial function after smoking cessation by using flow-mediated dilation (FMD) but did not observe this improvement using reactive hyperemia peripheral artery tonometry (RH-PAT). This indicates that there is a disagreement between the changes measured by FMD and natural logarithmic transformation of the reactive hyperemia index (Ln-RHI). One of the reasons for this disagreement is thought to be that increases in FMD and Ln-RHI after successful smoking cessation correlate differently with clinical variables, including BMI and weight gain after smoking cessation.

A number of methods to assess endothelial function have been investigated. Studies of brachial artery FMD were first reported in 1992 [1], and this method now the most widely used method in clinical research. RH-PAT, a newly developed method, was first reported in 2003 [2]. Both FMD and RH-PAT are based on the same principle of the reactive hyperemia phenomenon. This involves increased blood flow following a period of transient arterial occlusion and serves as an index of endothelium-dependent vasodilator function. In FMD, the brachial arterial diameter is measured by ultrasound before and after inducing reactive hyperemia to increase the shear stress on the arterial wall [1]. The increased shear stress produced by reactive hyperemia induces activation of endothelial nitric oxide (NO) synthase, leading to an increase in NO production in the endothelium. Therefore, FMD is a

Toru Kato

torumed3@gmail.com

1 Department of Clinical Research, National Hospital Organization Tochigi Medical Center, Tochigi, Japan noninvasive marker of local NO bioavailability in the endothelium. Semiautomated devices to measure FMD have been introduced to overcome technical limitations and are now available in clinical settings. However, FMD measurement requires appropriate training to ensure technical validation and good reproducibility. RH-PAT is another technique for measuring endothelial function and has recently become available in clinical settings. The measurement of RH-PAT is based on noninvasive measurement of pulsatile volume changes at the fingertip before and after reactive hyperemia using peripheral arterial tonometry [2]. The procedure for inducing reactive hyperemia is the same as that used in the measurement of FMD. PAT probes are placed on the index finger of each hand, and the postocclusion to preocclusion ratio is then calculated. The RH-PAT signals are corrected for measurements in the contralateral arm. The measurement of RH-PAT is less operator-dependent than that of FMD. Although FMD reflects endothelial function in the conduit arteries and RH-PAT reflects endothelial function in the peripheral resistance arteries, it has been shown that the two methods are not closely related [3]. While FMD and RH-PAT may not be interchangeable markers, several prospective studies have demonstrated that they are both independent predictors of future cardiovascular events [4], and both methods have acceptable reproducibility. The Framingham Heart Study reported no statistically significant relationship between signals obtained with RH-PAT and FMD, suggesting that these two methods reflect distinct aspects of vascular function [5]. Similar results were obtained in a recently published systematic review and meta-analysis [4]. This publication included several prospective studies that focused on the prognostic value of FMD and RH-PAT and updated the evidence for FMD and RH-PAT as predictors of cardiovascular events.

Both FMD and RH-PAT are used to assess endothelial function $[6,7]$ and are useful indicators for evaluating this 
function. However, the target blood vessels of the two methods are different. FMD is measured mainly in the brachial artery, while RH-PAT is measured by recording the finger arterial pulse wave amplitude and calculating the RHI. There are distinct differences in the physiology of the vascular beds measured by these two methods and in the response of the conduit and resistance vessels to reactive hyperemia. This raises the question as to which index is better for evaluating improvements in vascular endothelial function after successful smoking cessation. This is an important issue in clinical practice. In their study, Fukumoto et al. [3] attempted to determine which method was better for assessing improvement in vascular endothelial function after successful smoking cessation. To achieve this objective, they used simultaneous measurement of FMD and RH-PAT. This approach demonstrated a significant improvement in endothelial function assessed by FMD but not by RH-PAT. In addition, Spearman's rank correlation coefficient and the intraclass correlation coefficient did not show an agreement in the changes measured by FMD or Ln-RHI. The authors speculated that the reason for this discrepancy might be that FMD and Ln-RHI are associated with different clinical variables.

A study in a large, community-based cohort [5] showed that the RHI did not correlate significantly with FMD. This suggested that physiological variations in vessel sizes may cause divergent brachial and digital reactivity and that FMD and RH-PAT provide different information on vascular function. For example, FMD is particularly sensitive to impairment caused by risk factors such as age and hypertension, whereas the RHI is more sensitive to metabolic risk factors such as body mass index (BMI) and diabetes. Similar to our previous report [6], the present study showed that smoking cessation led to a significant increase in BMI. An increase in the Ln-RHI was also found to correlate inversely with baseline BMI. The lower digital hyperemic response in obese participants was consistent with impaired microvessel flow reserve, which may contribute to a reduction in blood flow supply associated with increased metabolic demands. These results indicate that smokers with a higher baseline BMI may be more likely to have a decrease in Ln-RHI after smoking cessation that is often accompanied by an increase in BMI.

In the present study, blood pressure (BP) increased after smoking cessation, with the increase in FMD correlating inversely with the change in systolic BP. In contrast, Ln-RHI correlated positively with the change in systolic BP. The authors speculated that the elevation in BP may have led to increased microvascular compliance due to changes in vascular tone or structure, which in turn led to higher hyperemic pulse amplitude in the digits. Therefore, the differential effects of changes in systolic BP may contribute, in part, to the disagreement observed between the changes in FMD and Ln-RHI after smoking cessation.

To the best of our knowledge, this was the first study to simultaneously assess endothelial function using FMD and RH-PAT before and after smoking cessation and to demonstrate different serial changes in endothelial function between these two types of assessment. The results of the study indicate that the effect of smoking cessation on vascular endothelial function may be evaluated better by FMD than by RH-PAT. In other words, the findings of the study suggested that smoking cessation had a greater effect on the endothelial function of conduit vessels than on that of peripheral resistance vessels. However, a prospective, large-scale study is needed to determine whether the improvement in FMD associated with smoking cessation prevents cardiovascular events such as acute coronary syndrome by improving endothelial function of the conduit vessels.

\section{Compliance with ethical standards}

Conflict of interest The authors declares that he has no conflict of interest.

Publisher's note Springer Nature remains neutral with regard to jurisdictional claims in published maps and institutional affiliations.

\section{References}

1. Celermajer DS, Sorensen KE, Gooch VM, Spiegelhalter DJ, Miller OI, Sullivan ID, et al. Non-invasive detection of endothelial dysfunction in children and adults at risk of atherosclerosis. Lancet. 1992;340:1111-5.

2. Kuvin JT, Patel AR, Sliney KA, Pandian NG, Sheffy J, Schnall RP, et al. Assessment of peripheral vascular endothelial function with finger arterial pulse wave amplitude. Am Heart J. 2003;146:168-74.

3. Fukumoto K, Takemoto Y, Norioka N, Takahashi K, Namikawa H, Tochino Y, et al. Predictors of the effects of smoking cessation on the endothelial function of conduit and digital vessels. Hypertens Res. 2020. https://doi.org/10.1038/s41440-020-0516-z. Online ahead of print.

4. Matsuzawa Y, Kwon TG, Lennon RJ, Lerman LO, Lerman A. Prognostic value of flow-mediated vasodilation in brachial artery and fingertip artery for cardiovascular events: a systematic review and meta-analysis. J Am Heart Assoc. 2015;4:e002270.

5. Hamburg NM, Palmisano J, Larson MG, Sullivan LM, Lehman BT, Vasan RS, et al. Relation of brachial and digital measures of vascular function in the community: the Framingham Heart Study. Hypertension. 2011;57:390-6.

6. Kato T, Umeda A, Miyagawa K, Takeda H, Adachi T, Toyoda S, et al. Varenicline-assisted smoking cessation decreases oxidative stress and restores endothelial function. Hypertens Res. 2014;37:655-6.

7. Xue C, Chen QZ, Bian L, Yin ZF, Xu ZJ, Zhang AL, et al. Effects of smoking cessation with nicotine replacement therapy on vascular endothelial function, arterial stiffness, and inflammation response in healthy smokers. Angiology. 2019;70:719-25. 